\title{
Endurecimiento superficial mediante tratamiento térmicos y anodizado de la aleación Ti-6A1-4U para implantes quirúrgicos
}

\author{
GIL, F.J.; FERNÁNDEZ, E.; ARCAS, R. Y PLANELL, J.A \\ E.T.S. Ingenieros Industriales de Barcelona. \\ Universidad Politécnics de Cataluña.
}

\section{RESUMEN}

Se han estudiado dós métodos de endurecimiento de las aleaciones de titanio como es la anodización electroquímica y el tratamiento térmico de revenido de la microestructura martensítica. Este endurecimiento de la aleación Ti-6A1-4V mejorará la resistencia al desgaste de estas aleaciones en sus aplicaciones para implantes quirúrgicos. La anodización se ha realizado para tres microestructuras diferentes de la aleación Ti-6A1-4V y aplicando diferentes diferencias de potencial. Los tratamientos térmicos de revenido se han realizado a diferentes temperaturas y tiempos de tratamiento.

\section{SUMMARY}

Two methods of the titanium aleation hardening have been studied, like the electrochemical anodization and the termical treatment of the martensitic microstructure assented. This hardening of the Ti-6AI-4v aleation will improve the resistance to the slow waste of this aleations when using them as applications in the surgical implants. The anodization has been done for three different microstructures of the aleation Ti-6Al-4V and using different potential differences. The assented termical treatments have done in different temperatures and treatment timmings.

\section{INTRODUCCIÓN}

Las aleaciones de titanio utilizadas en implantes ortopédicos, son resistentes a la corrosión y compatibles con el cuerpo humano debido a al película de óxido que se forma rápidamente en la superficie ${ }^{1}$. Cuando la aleación de Titanio se utiliza en articulaciones, se debe efectuar un tratamiento de superficies con el fin de provocar un endurecimiento para aumentar su resistencia al desgaste ${ }^{2}$.

Hay diferentes métodos utilizados para endurecer el titanio y sus aleaciones. El que permite obtener películas de óxido endurecedoras más delgadas es el proceso de anodización, llegando a espesores menores de 100 $\mathrm{nm}$, estas capas representan mejoras sustanciales de la resistencia al desgaste pero no pueden soportar elevadas cargas. La implantación iónica consigue la incorporación de iones đe carbono y/o nitrógeno, llegando a espe- sores superiores a centenas de nanómetros ${ }^{3}$. El endurecimiento se hace posible por la formación de una solución sólida o por formación de precipitados, aumentando no sólo la resitencia al desgaste sino también la resistencia a fatiga del material. La nutrición iónica de las aleaciones de titanio presenta colores oro característicos del nitruro de titanio 0-TiN y por debajo de éste se presenta una capa de mayor espesor de e-TiN que produce el endurecimiento y que puede tener un espesor desde 10 a $100 \mu \mathrm{m}$. En algunas aleaciones de titanio se pueden alcanzar valores de dureza superiores a las mil unidades Vickers. ${ }^{4-5}$

La deposición electroquímica o química de cromo o niquel y la técnica del PVD (deposición física en fase vapor) que puede recubrir el titanio con TiN, alcanzan espesores de 1 a $10 \mu \mathrm{m}$. Estas técnicas de deposición pueden ser generadas por evaporación con ayuda de un haz de electrones, pulverización catódica en corriente continua, pulverización catódica con aplicación de campos magnéticos y pulverización catódica a alta frecuencia, o por plasma. ${ }^{6-7}$

Otro tipo de tratamientos superficiales son los tratamientos termoquímicos de difusión que principalmente son dos métodos, la difusión gaseosa estanca y los baños de sales. Son técnicas muy poco utilizadas debido a que en el tratamiento no debe estar presente el oxígeno ya que en este forma unas capas de óxido que hacen impenetrable el paso de otros elementos. También se debe tener especial cuidado en la introducción de hidrógeno ya que se forman hidruros de titanio que fragilizan el material. Los elementos que normalmente se utilizan en este tipo de tratamientos son el carbono, nitrógeno y boro. Las durezas superficiales que se obtienen son del orden de $750 \mathrm{HV}$. Estos valores de dureza aumentan la resistencia al desgaste en un factor de $10 \mathrm{y}$ el coeficiente de fricción pasa de 0,32 a $0,18^{7-8}$.

En este trabajo, se han estudiado los aumentos de dureza que se obtienen en los procesos de anodizado estudiando la influencia de los potenciales aplicados y la microestructura del material. También se estudia el aumento de dureza a la estructura martensítica sometida a tratamientos térmicos de revenido a diferentes temperaturas y tiempos. Estas técnicas de endurecemiento son más sencillas, versátiles y en los que se requieren como veremos pequeños tiempos de tratamiento tanto en los procesos de anodización como en los de revenido. 
TABLA 1

RESULTADOS DEL TRATAMIENTO DE ANODIZACIÓN PARA LA MICROESTRUCTURA MILL-ANNEALED

\begin{tabular}{rcccc}
\hline Probeta & $\begin{array}{c}\text { Voftaje } \\
(\mathrm{V})\end{array}$ & Color & $\begin{array}{c}\text { Espesor } \\
(\mathrm{mm})\end{array}$ & $\begin{array}{c}\text { Dureza } \\
H V\end{array}$ \\
\hline 0 & 0 & metálico & - & 280 \\
1 & 10 & dorado & 30 & 313 \\
2 & 15 & ocre & 40 & 340 \\
3 & 20 & azul & 70 & 356 \\
4 & 25 & azul & 90 & 361 \\
5 & 30 & azul & 120 & 369 \\
6 & 35 & verde & 150 & 372 \\
7 & 40 & verde & 170 & 375 \\
8 & 45 & verde & 240 & 380 \\
9 & 50 & amarillo & 290 & 383 \\
10 & 55 & amarillo & 320 & 385 \\
11 & 60 & violeta & 420 & 393 \\
\hline
\end{tabular}

TABLA 2

RESULTADOS DEL TRATAMIENTO DE ANODIZACIÓN PARA LA MICROESTRUCTURA WIDMANSTATTEN

\begin{tabular}{rcccc}
\hline Probeta & $\begin{array}{c}\text { Voltaje } \\
(\mathrm{V})\end{array}$ & Color & $\begin{array}{c}\text { Espesor } \\
(\mathrm{mm})\end{array}$ & $\begin{array}{c}\text { Dureza } \\
H V\end{array}$ \\
\hline 0 & 0 & metálico & - & 319 \\
1 & 10 & dorado & 20 & 335 \\
2 & 15 & ocre & 30 & 360 \\
3 & 20 & azul & 70 & 370 \\
4 & 25 & azul & 80 & 375 \\
5 & 30 & azul & 80 & 390 \\
6 & 35 & verde & 140 & 392 \\
7 & 40 & verde & 140 & 400 \\
8 & 45 & verde & 180 & 410 \\
9 & 50 & amarillo & 220 & 412 \\
10 & 55 & amarillo & 240 & 415 \\
11 & 60 & violeta & 360 & 421 \\
\hline
\end{tabular}

TABLA 3

RESULTADOS DEL TRATAMIENTO DE ANODIZACIÓN PARA LA MICROESTRUCTURA MARTENSITICA

\begin{tabular}{ccccc}
\hline Probeta & $\begin{array}{c}\text { Voltaje } \\
(\mathrm{V})\end{array}$ & Color & $\begin{array}{c}\text { Espesor } \\
(\mathrm{mm})\end{array}$ & $\begin{array}{c}\text { Dureza } \\
H V\end{array}$ \\
\hline 0 & 0 & metálico & - & 330 \\
1 & 10 & dorado & 30 & 430 \\
2 & 15 & ocre & 30 & 440 \\
3 & 20 & azul & 70 & 440 \\
4 & 25 & azul & 80 & 455 \\
5 & 30 & azul & 80 & 442 \\
6 & 35 & verde & 140 & 445 \\
7 & 40 & verde & 160 & 445 \\
8 & 45 & verde & 180 & 443 \\
9 & 50 & amarillo & 250 & 460 \\
10 & 55 & amarillo & 260 & 460 \\
11 & 60 & violeta & 500 & 455 \\
\hline
\end{tabular}

\section{MATERIALES Y MÉTODO EXPERIMENTAL}

La aleación Ti-6A1-4V utilizada en el presente trabajo fue gentilmente donada por Traiber S.A., presentando una composición química con bajos contenidos en elementos intersticiales y una microestructura de recepción correspondiente a la «mill annealed», cumpliendo la norma ASTM F136-84 para el Ti-6A1-4V forjado para aplicaciones quirúrgicas.

\section{Tratamiento de Anodización}

E1 proceso electrolítico se realiza con una solución acuosa de sulfatos amónico y magnésico, ortofosfato sódico y ácido sulfúrico con ánodo de acero inoxidable. $\mathrm{La}$ anodización se realiza a potenciales diferentes manteniéndose el tiempo constante a $10 \mathrm{~s}$, obteniéndose capas de oxidos de diferentes estequiometrías y grosores. El espesor de estas capas fue determinado por un medidor de espesor de alta sensibilidad mediante la aplicación de corrientes de Foucault. Se realizaron ensayos de dureza Vickers con cargas de $1000 \mathrm{~g}$ y se determinan los valores en función del potencial aplicado.

Este tratamiento se ha realizado a probetas pulidas con estructura de recepción («mill-annealed») y a probetas en las cuales se ha realizado un tratamiento térmico durante 1 hora en atmósfera de Argón, a temperaturas superiores de la $\beta$-transus, que paras esta aleación es de $1040^{\circ} \mathrm{C}$. Posteriormente, unas se enfriaron en el interior del horno obteniendo unas microestructuras de placas $\alpha$ de Widmanstatten con fase $\beta$ rodeando dichas placas y las restantes, se templaron en agua a $20^{\circ} \mathrm{C}$, resultando una estructura martensítica $\alpha$ de morfología acicular.

\section{Tratamiento térmico de revenido}

Las probetas con microestructura martensítica se sometieron a un tratamiento térmico de revenido a 400 , 700 y $800{ }^{\circ} \mathrm{C}$ durante diferentes tiempos de tratamiento con temple posterior en agua a $20^{\circ} \mathrm{C}$. Dichos tratamientos térmicos fueron llevados a cabo mediante atmósfera controlada de Argón.

Las muestras se prepararon metalográficamente y se determinaron para cada una su dureza Vickers aplicando $1000 \mathrm{~g}$ de carga durante 15 segundos. Este ensayo se realizó en los cortes longitudinales y transversales debido a que la incorporación de poca cantidad de átomos de oxígeno producen variaciones significativas en los valores de dureza.

\section{RESULTADOS}

Los resultados del tratamiento de anodización se muestran en las Tablas 1, 2 y 3 correspondientes a las microestructuras de mill annealed, Widmanstatten y martensítica, respectivamente.

\section{DISCUSIÓN}

Estos tratamientos de anodización producen aumentos de unas cien unidades Vickers para cada microestructu- 
ra en las condiciones de 60 Voltios de diferencia de potencial y diez segundos en el tiempo de aplicación. Si relacionamos el valor de dureza de la mill annealed con el máximo obtenido para la microestructura martensítica se obtienen aumentos del orden del $60 \%$.

Además de este aumento de dureza superficial que se traducirá con un aumento en la resistencia al desgaste ${ }^{9}$, estas capas de óxido de Titanio obtenidas producen un descenso en la liberación de iones titanio, aluminio y vanadio al cuerpo humano. Este hecho, hace que desciendan los riesgos de irritaciones locales o metalosis a largo plazo debido a la difusión de iones metálicos en los tejidos circundantes ${ }^{10}$.

Los resultados de dureza respecto al tiempo de revenido se observan en la figura 1 . Las formas de las curvas es propia de procesos de nucleación y crecimiento. Se aprecia para los tratamientos a $800^{\circ} \mathrm{C}$ un brusco aumento de los valores de dureza hasta unos 20 minutos de tratamiento, para posteriormente estabilizarse a valores de unos $410 \mathrm{HV}$. Para el tratamiento realizado a $700{ }^{\circ} \mathrm{C}$ se obtiene menores produciéndose la estabilización de dureza en un tiempo de unos 30 minutos. Para los tratamientos a temperaturas de $400{ }^{\circ} \mathrm{C}$ se observa un incremento muy pequeño de dureza, obteniéndose unos valores máximos de $350 \mathrm{HV}$.

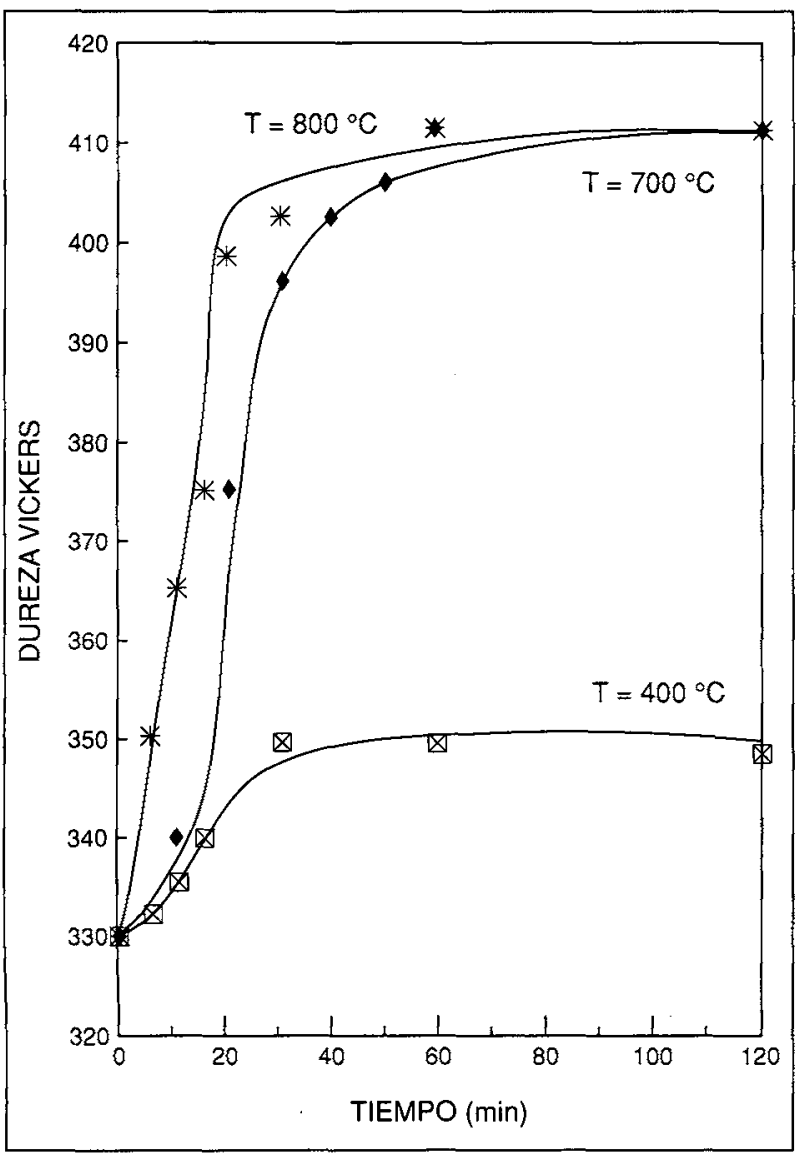

Figura 1: Variaciones de dureza con el tiempo revenido para diferentes temperaturas de tratamiento.
El endurecimiento producido por el tratamiento térmico de revenido se debe a la descomposición de la estructura martensítica $\alpha$ en fases de equilibrio $\alpha$ y $\beta$ que quedan como precipitados en la interfacie de las placas martensíticas que se van descomponiendo. ${ }^{11-12}$

Por los resultados parece que los niveles máximos de dureza que se alcanzan por estos tratamientos son los $410 \mathrm{HV}$ para las temperaturas de 800 y $700{ }^{\circ} \mathrm{C}$ ensayadas. La mayor rápidez de conseguir estos valores de dureza en la temperatura de $800{ }^{\circ} \mathrm{C}$ es debido a que a mayor temperatura la difusión atómica que origina la precipitación es más rápida. Para las temperaturas de $400{ }^{\circ} \mathrm{C}$ no se alcanzan estos valores de dureza debido a que al ser una temperatura menor la precipitación está menos favorecida.

Los valores de $410 \mathrm{HV}$ corresponden a un aumento en los niveles de dureza cercano a un $50 \%$ con relación a la microestructura original de «mill annealed».

Estas técnicas de endurecimiento están en fase de estudio para alcanzar mayores valores de dureza ya que los valores obtenidos son claramente esperanzadores para en un futuro poderse aplicar en prótesis de cadera y rodilla, especialmente.

\section{AGRADECIMIENTOS}

Los autores desean agradecer la donación del material del presente estudio a TRAIBER S.A., a la CICYT por la concesión del proyecto de investigación MAT 90 0755 y a la Universidad Politécnica de Cataluña por la concesión del proyecto FIU1922 que ha sufragado en parte este trabajo.

\section{BIBLIOGRAFIA}

1. Semlitsch M. Titanium alloy for hip joint replacements. Clinical Materials 1, 1-3 (1987)

2. Galante J.O., Rostoker W. Wear in total hip prostheses. Acta Orthop. Scand. Suppl. 145, 1-46 (1973).

3. Elder J.E., Thamboraj R. y Patraik (P.C). «Optimisation des conatianas d'une implantation d'ions pour améliorar les résistances a l'usure, à la fatigue de contact de l'alliage Ti6A14V»! International Materials Reviews.

4. Lanagan J., Morton P.M., Bell T.«Traitement de surface du titane avec un plasma à décharge luminiscente». Designing with Titanium (91986). Institute of Metals. 136-150.

5. Bacci T., Pradelli G., Besi B., Gianoglio C., Bandini C. «Surface engineering and chemical characterization in ion-nitride titanium and titanium alloys». Journal of Materials Science 25. 43094314. (1990).

6. Bell T., European J. Engineering Education, 12. 27-32. (1987).

7. Finern B. Harterei Techniscle Mitteilungen 26. 47-52. (1971).

8. Morton P.H. y Bell T. «Les procédés technologiques de surface appliqués au titane». Traitement Thermique 2. 241-290. (1990).

9. Kossovsky R., y Wei W. "Friction and War". Surface Modification Engineering. Vol.I. CRC press. 145-155 (1988).

10. Cigada A., Cabrini M., Pedeferri P. «Increasing of the corrosion resistance of the Ti-6A1-4V alloy by high thickness anodic oxidation». Journal of Materials Science Materials in Medicine. 3. 408-412 (1992)

11. Polmear I.J. «Light alloys». Edward Arnold. Londres.(1981).

12. Collings E.W. «The physical metallurgy of Titanium Alloys». American Society for Metals. Ohio. (1984). 